\title{
Studies to evaluate the outcome of DM in the public and private sector in China
}

\author{
Karen Y. L. Lo-Hui \\ Hong Kong Workers' Health Centre, Hong Kong SAR, China. karen@hkwhc.org.hk
}

Disability management (DM) is quite a fresh idea to Mainland China (Mainland). The government has thus turned to professionals from outside Mainland, i.e. Hong Kong (HK). Nevertheless, since HK is under 'one country, two systems' policy, it has developed an approach differing from that of Mainland. A DM pilot study was jointly conducted by the Guangdong Provincial Work Injury Rehabilitation Center (GPWIRC) and the Hong Kong Workers' Health Centre (HKWHC) to review the developments of DM reform in China and HK. In China, the foundation of DM approach is the work injury insurance system. Under this system, GPWIRC established her services to provide work injury prevention and occupational rehabilitation as a pilot study in early 2000s. Following this pilot project, GPWIRC further develops work and social rehabilitation and work injury prevention in the context of the labor insurance system. While in HK, mainly NGOs, insurance companies and some public organizations contribute to the promotion of DM approach. On the other hand, the study also reveals similar challenges that Mainland and HK are currently facing in the development of DM, such as professional's training in local rehabilitation, the underdeveloped reimbursement system and etc.. The aforementioned pilot study shows that DM's principles are accepted at a national level and some specific public organizations in China context. There thus is a research need to study the current DM development situation in the private sector. By doing so, an ongoing study, namely "Demographic change and private sector disability management in Australia, Canada, China and Switzerland. A comparative study" launched in Nov. 2013. Through this research, questions of process and procedure of the DM system used in the company, benefits gained and drawbacks encountered by the companies, and the strengths and weaknesses in the current DM systems will be answered. Other than that, data will also be collected from the employees' perspective on their job satisfaction, physical and mental health, employee morale and workplace attendance and etc.. The primary result is expected in 2016. 